\title{
Uma classificação de tipos de objetos de patrimônio para integração de acervos digitais de arquivos, bibliotecas e museus
}

\author{
Una clasificación de los tipos de objetos patrimoniales para la integración \\ de colecciones de archivos digitales, bibliotecas y museos
}

A classification of heritage object types to integrate the digital collections of archives, libraries and museums

\section{Carlos Henrique MARCONDES}

PPGCI/UFF - Programa de Pós-Graduação em Ciência da Informação da Universidade Federal Fluminense, Rua Tiradentes 148, Ingá - Niterói - RJ - Brasil - CEP: 24210-510, ch_marcondes@id.uff.br

\begin{abstract}
Resumen
Se propone una clasificación exhaustiva de los tipos de objetos existentes en las colecciones de instituciones de memoria y cultura, que puede favorecer la navegación, el descubrimiento, la integración y la interoperabilidad entre dichas colecciones.
\end{abstract}

Palabras clave: Colecciones digitales. Clasificación. Instituciones de memoria y cultura. Acceso integrado. Interoperabilidad. Búsqueda federada. Información del patrimonio. Búsqueda facetada. Brasil.

\section{Introdução}

A partir do surgimento da Web as instituições de Memória e Cultura - arquivos, bibliotecas e museus -, vêm digitalizando seus acervos e os disponibilizando na Web através de ferramentas informatizadas como os catálogos. Os catálogos específicos de cada instituição se constituem em "silos de dados" (Fink, 2018), que restringem as possibilidades de acesso e integração desses acervos somente ao ambiente tecnológico específico de cada catálogo. Mais recentemente, com a emergência das tecnologias LOD (Bizer et al., 2009) - linked open data - dados abertos interligados, registros digitais dos objetos dos acervos podem ser publicados diretamente na Web, com "links" próprios, como conteúdos estruturados, legíveis por máquinas e não somente por pessoas, como as páginas hipertextuais da Web convencional; além de publicados esses registros podem também ser consultados conjuntamente, formando um grande banco de dados independente de suas instituições de origem, passando a constituir "Linked Open Data Cloud", ou nuvem de dados em Memória e Cultura.

Portais Web são o mecanismo mais comum de acesso integrado a acervos heterogêneos, originários de diferentes instituições (Marcondes, 2017). Exemplos são a Biblioteca Europeana (1), a DPLA - Digital Public Library of America (2), o

\begin{abstract}
A comprehensive classification of the types of objects existing in the collections of the institutions of memory and culture, which may enable navigation, discovery, integration and interoperability among such collections.
\end{abstract}

Keywords: Digital collections. Classification. Memory and culture institutions. Integrated access. Interoperability. Federated searching. Heritage information. Faceted searching. Brazil.

portal TROVE da National Library of Australia (3), Hispaña (4) - Acceso en Linea al Patrimonio Cultural -, a Mexicana - Repositório del Patrimonio Cultural de Mexico (5) e, no Brasil a Brasiliana Iconográfica (6) e o portal da Rede Web de Museus (7). $O$ acesso através desses portais integrados ou de registros publicados segundo as tecnologias LOD permite que sinergias, superposições e complementaridades possam ser exploradas, permitindo "trajetos", "visões", "perspectivas" e "modos" específicos para navegar pelos acervos; tornam-se viáveis exposições virtuais ou recursos educacionais online integrando objetos digitais de acervos de diferentes instituições.

Portais deste tipo dão acesso a materiais bastante heterogêneos, quanto ao gênero, tipologia, temática, formato, idioma, relação com pessoas ou períodos históricos, estilos artísticos, etc., além de serem originários de diferentes instituições. Estamos no mundo da Cultura e não de recursos especializados tematicamente. Ao mesmo tempo em que essa heterogeneidade maximiza o valor cultural e educativo destes acervos, torna complexo o projeto das interfaces de acesso. São recursos informacionais de diversas temáticas, não contam com um vocabulário que padronize e especifique a terminologia a ser utilizada nas buscas. Atualmente projetos de interfaces a sistemas de gestão de acervos cultu- 
rais seguem as diretrizes chamadas de "Generous Interfaces" (8), na qual caixas de busca, onde o usuário digita as palavras-chave, são substituídas por interfaces que procuram mostrar a priori os conteúdos existentes nos sistemas; a simples caixa de busca por palavras-chaves, segundo esta concepção, na verdade é uma "caixapreta" que não permite que usuários se orientem sobre os conteúdos disponíveis (Whitelaw, 2015). Uma interface projetada segundo esses princípios deve prover diferentes taxonomias ou vocabulários específicos de navegação (Marcondes, Souza, 2018), que permitam aos usuários ter uma visão dos conteúdos do sistema, se orientar e se acercar dos conteúdos desejados.

Neste contexto, para permitir a exploração e navegação por acervos digitais heterogêneos, tornase importante o desenvolvimento de vocabulários que expressem as diferentes facetas (Broughton, 2006), (Ménard, Mas e Alberts, 2010), (Sinclair et al 2005) ou aspectos através dos quais objetos de Memória e Cultura possam ser acessados; entre estas estão, no caso brasileiro, períodos históricos, estilos/movimentos artísticos, técnicas, eventos ou pessoas relacionadas a objetos de Memória e Cultura, grupos étnicos formadores do povo brasileiro, e mesmo relacionamentos culturalmente significativos entre objetos de diferentes acervos (Marcondes, 2018a), etc.

Ao publicar acervos na Web os objetos digitais que os constituem são representações dos diferentes tipos de objetos de Memória e Cultura. Qualquer descrição estruturada de objetos de Memória e Cultura ao ser patromonializado e descrito em uma instituição se inicia por assinalar a esse objeto um identificador e um tipo. Assim uma faceta existente em todo objeto de Memória e Cultura seria o que esse objeto é, sua natureza ontológica, seu tipo; em documentação, sua Classificação, Categoria, Tipo de Objeto, Nome do Objeto, etc. Um vocabulário estruturante para integração de acervos digitais de Memória e Cultura seria, portanto, uma classificação abrangente de todos os tipos de objetos que possam existir em acervos de arquivos, bibliotecas, museus, além de outras instituições. Tal classificação seria capaz de integrar e favorecer a interoperabilidade entre diferentes acervos ao permitir a usuários explorar e navegar transversalmente recuperando objetos do mesmo tipo, pertencentes a acervos diferentes.

\section{Objetivos}

Este trabalho tem como objetivo propor uma classificação abrangente de tipos de objetos existentes em acervos de instituições de Memória e Cultura, que possa favorecer a navegação, descoberta, integração e interoperabilidade entre esses acervos. O trabalho esta assim organizado: a seção 3 apresenta as questões que motivaram a proposta, a seção 4 descreve o material e métodos utilizados, a seção 5 apresenta e discute os resultados e a seção 6 apresenta conclusões da pesquisa.

\section{Motivação}

O trabalho foi motivado originalmente (Marcondes et al, 2016) pelas necessidades da Rede Web de Museus do Estado do Rio de Janeiro, que utilizava o Thesaurus para Acervos Museológicos (Ferrez e Bianchini, 1987) para integrar diferentes acervos. O Thesaurus, largamente utilizada por instituições museológicas por todo o Brasil, é uma classificação com 16 categorias, todas de objetos de tipo artefato. Suas 16 categorias são: 1. Caça e Guerra, 2. Artes Visuais, 3. Objetos Pecuniários, 4. Construção, 5. Interiores, 6. Trabalho, 7. Lazer, 8. Insígnias, 9. Objetos Cerimoniais, 10. Comunicação, 11. Transporte, 12. Objetos pessoais, 13. Castigo/Penitência, 14. Medição/Registro, 15. Embalagens, 16. Amostras/Fragmentos.

Como parte de sua política de desenvolvimento, a Rede Web passou a integrar museus com coleções de fósseis, mineralogia, botânica (ex: Museu Nacional do Rio de Janeiro, Brasil), e outros, de patrimônio cultural imaterial, as quais não tinham categorias correspondentes no Thesaurus (Ferrez e Bianchini, 1987). Houve então a necessidade de estendê-lo para abranger esse tipo de acervo.

As motivações atuais são duas: a necessidade de integrar e dar interoperabilidade entre acervos digitais de arquivos, bibliotecas e museus brasileiros quanto acessados através de portais Web; e a proposição de um vocabulário de relacionamentos culturalmente significativos (Marcondes, 2018a; 2018b) entre objetos de diferentes acervos; para formalizar esse vocabulário é necessário especificar, para cada tipo de relacionamento, seu domínio e escopo em termos de tipos de objetos de Memória e Cultura. Foi necessário então criar uma classificação abrangente de tipos de objetos de Memória e Cultura.

\section{Material e método}

A literatura, os diferentes esquemas de classificação (British Museum Materials Thesauros, 1997; Collections Trust, Terminologies; The Getty Research Institute, Art \& Architecture Thesaurus; Heritage, Canada, 2016) e os modelos conceituais de objetos de patrimônio (Europeana, 2017; The CIDOC Conceptual Reference Model, 2014; IFLA, 1995; International Council On Archives, Experts Group On Archival Description, 2016) foram utilizados como fontes para categorias que pudessem 
subsumir as dezesseis originais do Thesaurus e pudessem incluir coleções de patrimônio cultural imaterial e científico. Definições de objeto patrimonializado (Brier, 2016; Merlot, 2004), artefato, objeto natural, entre outras, também foram coletadas. As definições de manifestações do patrimônio cultural imaterial foram coletadas de documentos oficiais relativos à política brasileira de patrimônio cultural imaterial (Cavalcanti e Fonseca, 2008). Essas definições foram usadas como insumos para definir classes de entidades. Análise ontológica (Guarino, 1995), (Guarino e Welthy, 2000). A Teoria processo de construção social da realidade de Searle (1995), definições conceituais (Dahlberg, 1981) e teoria de classificação aristotélica (Berg, 1982) foram então utilizadas como fundamentação teórica para analisar, distinguir e identificar classes disjuntas de objetos de patrimônio e para integrá-los em um esquema classificatório geral. Usou-se um procedimento botton up a partir das 15 categorias do Thesaurus, para gerar novas categorias, tentando responder às questões: que categoria subsumiria as 15 do Thesaurus (exceto a categoria 16. Amostras/Fragmentos)? Existiriam outros tipos de objetos patrimonializados que estariam excluídos da categoria assim gerada?

O esquema classificatório proposto é apresentado em uma Tabela com 6 colunas, sendo as 5 primeiras para os 5 níveis de profundidade do esquema e a $6^{a}$ coluna para "links" para possíveis categorias em esquemas compatíveis com as tecnologias LOD, ou seja, esquemas abertos, que permitem o reuso amplamente. No Anexo 1 o esquema classificatório proposto é apresentado usando SKOS (2009) - Simple Knowledge Organization System -, para que possa ser reusado. O SKOS é um padrão proposto pelo W3C para a representação de sistemas de organização do conhecimento como taxonomias, thesaurus, etc., compatível com as tecnologias LOD. A versão SKOS foi gerada usando a ferramenta de software TemaTres (8).

\section{Resultados e discussão}

O que seriam as 15 categorias de objetos do Thesaurus (Ferrez e Bianchini, 1987)? De acordo com as definições coletadas (Baker, 2004, p. 99; Borgo et al, 2014, p. 1; Hilpinen, 2011), são todas artefatos materiais, poderiam ser subsumidas a uma categoria com essa denominação.

A partir dessa categoria, Artefatos Materiais, operou-se pela análise ontológica baseada nas diferenças, ou exclusões, o que é chamado por Guarino $(1995$, p. 2) ao se referir a Ontologia como "intended as a theory of a priori distinctions". Como consequência a questão a ser respondida seria: Que objetos não são Artefatos Materiais?
A resposta produziu uma nova categoria, Objetos de Cultura Imaterial. A UNESCO fornece diferentes definições e enumera exemplos de manifestações de Cultura Imaterial, como a seguinte:

Intangible or immaterial cultural heritage encompasses life expressions and traditions that communities, groups and people from all over the world inherit from their ancestors and pass their knowledge to their descendants. Besides sound and video recording, and archives, UNESCO considers that one of the most effective ways of preserving intangible heritage is to ensure that the bearers of this heritage can continue producing it and transmitting it.

A essa categoria estaria subsumida a categoria Objetos de Cultura Popular Imaterial.

Buscou-se também definições para as chamadas artes performáticas (Wikipedia). Estas são formas artísticas processuais, cada manifestação é uma performance específica. Uma performance só pode ser patrimonializada ao ser registrada. Estão ai incluídas teatro, ballet, música, ópera, canto, entre outras (Doerr, Bekiari e LeBoeuf, 2008).

A categoria que subsumiria Artefatos Materiais e Objetos de Cultura Imaterial seria a de Produtos da Cultura Humana.

Restaria uma outra oposição a ser explorada, a dos objetos patrimonializados que não são produtos da cultura humana. De fato, existem inúmeras instituições de Memória e Cultura que mantém coleções de objetos que não são produtos da cultura humana, são (ou foram) objetos naturais; são herbários, jardins zoológicos, aquários, museus de história natural, coleções entomológicas, paleontológicas, de mineralogia, etc. O Britsh Museum Materials Thesaurus tem como categorias mais amplas "Organic", "Inorganic" e "Processed Material"; no entanto, a categoria "Inorganic Material" contempla a faceta "materiais" e não artefatos.

Todos esses constituiriam então a categoria de Objetos Naturais, por sua vez subdivididos em Objetos (originariamente) Inorgânicos, como coleções de mineralogia, e Objetos Orgânicos ou originariamente orgânicos: fósseis, coleções entomológicas, amostras botânicas ou zoológicas, etc.

$\mathrm{E}$ a categoria mais geral? O que são objetos de patrimônio? A definição de Stransky (1985, p. 98) para objetos museológicos serviu de base para a definição adotada: objetos de patrimônio são "ontologically coincident with objects in general, but as to their semantic, they have a new function, i.e. the function of authentic witnesses, documents, and/or the testimony of natural and social facts".

A classificação final proposta é a que se segue (Tabela I). Na $6^{a}$ coluna "aat": significa Arts \& Architecture Thesaurus e "crm": significa CIDOC CRM Conceptual Model. 


\begin{tabular}{|c|c|c|c|c|c|}
\hline $1^{\circ}$. nível & $2^{\circ}$. nível & $3^{\circ}$. nivel & $4^{\circ}$. nível & $5^{\circ}$. nível & Links p. outros vocabulários \\
\hline \multicolumn{6}{|c|}{$\begin{array}{l}\text { Objetos } \\
\text { (patrimonializados) } \\
\text { de Memória e } \\
\text { Cultura }\end{array}$} \\
\hline & $\begin{array}{l}\text { Objetos naturais } \\
\text { (originariamente) }\end{array}$ & & & & $\begin{array}{l}\text { Ver Wikidata "Nature" } \\
\text { https://www.wikidata.org/wiki/ } \\
\text { Q7860 }\end{array}$ \\
\hline & & $\begin{array}{l}\text { Objetos } \\
\text { inorgânicos } \\
\text { (originariamente) }\end{array}$ & & & $\begin{array}{l}\text { Ver Wikidata "Inorganic } \\
\text { chemistry" } \\
\text { https://www.wikidata.org/wiki/ } \\
\text { Q11165 }\end{array}$ \\
\hline & & $\begin{array}{l}\text { Objetos orgânicos } \\
\text { (originariamente) }\end{array}$ & & & $\begin{array}{l}\text { Ver Wikidata "Life" } \\
\text { https://www.wikidata.org/wiki/ } \\
\text { Q3 }\end{array}$ \\
\hline & $\begin{array}{l}\text { Produtos da } \\
\text { Cultura Humana - } \\
\text { objetos feitos pelo } \\
\text { homem }\end{array}$ & & & & $\begin{array}{l}\text { crm:E } 71 \text { Man-made thing } \\
\text { http://www.cidoc- } \\
\text { crm.org/html/5.0.4/cidoc- } \\
\text { crm.html\#E71 }\end{array}$ \\
\hline & & $\begin{array}{l}\text { Objetos de cultura } \\
\text { material ou }\end{array}$ & & & $\begin{array}{l}\text { aat: http://vocab.getty.edu/aat/ } \\
300386957\end{array}$ \\
\hline & & Artefatos & & & $\begin{array}{l}\text { crm: E } 24 \text { Physical Man-made } \\
\text { thing } \\
\text { http://www.cidoc- } \\
\text { crm.org/html/5.0.4/cidoc- } \\
\text { crm.html\#E24 }\end{array}$ \\
\hline & & & $\begin{array}{l}\text { Documentos } \\
\text { textuais }\end{array}$ & & $\begin{array}{l}\text { aat: http://vocab.getty.edu/aat/ } \\
300263751\end{array}$ \\
\hline & & & & Manuscritos & $\begin{array}{l}\text { aat: } \\
\text { http://vocab.getty.edu//aat/ } \\
300028569\end{array}$ \\
\hline & & & & $\begin{array}{l}\text { Impressos } \\
\text { (livros, } \\
\text { periódicos, } \\
\text { etc., materiais } \\
\text { típicos de uma } \\
\text { biblioteca }\end{array}$ & $\begin{array}{l}\text { "Printouts", att: } \\
\text { http://vocab.getty.edu/aat/ } \\
300028467\end{array}$ \\
\hline & & $\begin{array}{l}\text { Objetos de cultura } \\
\text { imaterial (seus } \\
\text { registros) }\end{array}$ & & & \\
\hline & & & $\begin{array}{l}\text { Objetos } \\
\text { performáticos } \\
\text { da Cultura } \\
\text { popular }\end{array}$ & & \\
\hline & & & $\begin{array}{l}\text { Objetos das } \\
\text { Artes } \\
\text { performáticas }\end{array}$ & & $\begin{array}{l}\text { Wikidata: } \\
\text { https://www.wikidata.org/wiki/ } \\
\text { Q184485 }\end{array}$ \\
\hline
\end{tabular}

Tabela I. Classificação dos Tipos de Objetos de Patrimônio

Sob a categoria Objetos de cultura material também deveriam estar objetos de Memória e Cultura que formam os acervos de arquivos e bibliotecas. "Documento" e "Livro" estão incluídos na Categoria 10 Comunicação da versão original do Thesaurus (Ferrez, Bianchini, 1987, 60, 61), mas não na versão atualizada, o Tesauro de Objetos do Patrimônio Cultural nos Museus Brasileiros. Sugerimos a categoria Documentos textuais.

Embora tenha sido pesquisado em instituições como IFLA e ALA, não foi encontrada nenhuma classificação padronizada dos tipos de objetos que compõe o acervo de bibliotecas, embora haja muita coincidência nesta tipologia. A maioria das tipologias de materiais de bibliotecas encontradas (9) relaciona materiais como livros, periódicos, jornais, material de referência (dicionários, enciclopédias), publicações oficiais, teses e dissertações, mapas, manuscritos, obras raras, etc., coleções especiais (partituras musicais, material sonoro, filmes e vídeos) entre outros. Como já foi mencionado, sob essa categoria se encontrariam as tipologias ou convenções adotadas por bibliotecas específicas. No entanto, até onde podemos verificar, todas são tipologias de tipos de objetos, mantendo assim o princípio de coerência do esquema.

A presente proposta teve origem e foi pensada para ser utilizada em sistemas em rede, como 
acervos heterogêneos, originários de diferentes instituições. Uma vez estabelecido este esquema organizador, as subcategorias a serem desdobradas dependerão de decisões pragmáticas e políticas das instituições que estejam compartilhando seus acervos. Como esta pesquisa constatou, a grande maioria dos tesauros, classificações e vocabulários utilizados em instituições de Memória Cultura foi concebida para utilização em um tipo específico de acervo ou instituição.

A inclusão de diferentes tesauros ou classificações poderia ser dar por subcategorias específicas subordinadas à categoria Objetos de cultura material. Exemplos, no caso brasileiro, seriam o Tesauro de Cultura Material dos Índios no Brasil (Motta, 2006), do Museu do Índio, Rio de Janeiro, Brasil, que poderia ser integrado pela sua faceta Artefato; ou ainda o Tesauro de Folclore e Cultura Popular, do Museu de Folclore Edison Carneiro, Rio de Janeiro, Brasil pela sua categoria Artefato; ou o caso do Thesaurus de Acervos Científicos em língua portuguesa, desenvolvido pelo MAST Museu de Astronomia e Ciências Afins, Rio de Janeiro, Brasil - em cooperação com museus portugueses (10), que poderia ser integrado ao esquema proposto por todas as suas Categorias, que são todas objetos: Instrumento científico, Instrumento de demonstração e estudo, Máquina, Objeto de referência e Utensílio.

\begin{tabular}{ll}
\hline dc:title & $\begin{array}{l}\text { El ingenioso hidalgo don Quijote de la } \\
\text { Mancha, 2a parte }\end{array}$ \\
\hline dc:creator & Cervantes Saavedra, Miguel \\
\hline dc:contribuitor & Juan de la Cuesta \\
\hline dc:date & 1615 \\
\hline dc:type & http://localhost/tematres3.0/vocab/?tema=9 \\
\hline dc:type & "Printed documents" \\
\hline dc:identifier & http://bdh.bne.es/bnesearch/detalle/bdh00 \\
& 00047955 \\
\hline dc:publisher & BNE \\
\hline
\end{tabular}

Tabela II. Registro DC utilizando a classificação proposta no elemento dc:type

Como pode ser observado na Tabela I, coluna "Links p. outros vocabulários" (que contém "links" para conceitos de vocabulários que são compatíveis com as tecnologias LOD), estes se concentram nas categorias "Produtos da Cultura Humana", "Documentos textuais" e principalmente "Objetos de cultura material ou Artefatos". É ai que se concentram as propostas mais gerais de classificar objetos, embora existam vocabulários compatíveis com as tecnologias LOD mais específi- cos, como o AAT. Por isso tentou-se complementar esta ausência com "links" para entradas possivelmente pertinentes da Wikidata, que é um recurso curado compatível com as tecnologias LOD.

A seguir é apresentado, à guisa de exemplo, um registro da obra Don Quijote de La Mancha $2^{\mathrm{a}}$. parte, de Miguel de Cervantes Saavedra, codificado usando o vocabulário DC - Dublin Core - e utilizando o esquema classificatório proposto. O tipo de objeto segundo o esquema proposto é registrado no elemento dc:type.

\section{Conclusões}

Propusemos aqui, através da análise de diversos esquemas classificatórios e thesaurus de objetos de patrimônio, da análise ontológica, das definições conceituais, um esquema classificatório para objetos de patrimônio que subsumisse $e$ fosse mais geral que os esquemas classificatórios centrados na categoria "artefatos". O esquema proposto comporta objetos de patrimônio de coleções típicas de arquivos, bibliotecas e museus. O esquema permite também que classificações especializadas em tipos específicos de objetos possam ser integradas, subsumidas às categorias propostas, como foi mostrado na seção anterior.

Uma classificação geral de tipos de objetos de patrimônio é cada vez mais necessária, a medida desenvolvimento das tecnologias permite a integração de acervos digitais heterogêneos, criando novas e inusitadas potencialidades. Essa seria uma classificação integradora, à medida que todas as instituições de patrimônio mantem acervos de objetos. O esquema classificatório aqui apresentado é uma proposta inicial, com o objetivo primeiro de ser discutido com a comunidade dos gestores de coleções de patrimônio na direção de um esquema que posas favorecer a interoperabilidade e integração entre os diferentes acervos digitais de memória e patrimônio.

\section{Apoios}

O presente trabalho foi realizado com apoio da Coordenação de Aperfeiçoamento de Pessoal de Nível Superior - Brasil (CAPES) - Código de Financiamento 001 e do CNPq, processo $305253 / 2017-4$. Agradecemos também as valiosas contribuições dos revisores.

\section{Notas}

(1) https://www.europeana.eu/portal/pt

(2) https://dp.la/

(3) https://trove.nla.gov.au/

(4) http://hispana.mcu.es

(5) https://mexicana.cultura.gob.mx/ 
(6) https://www.brasilianaiconografica.art.br/

(7) http://www.museusdoestado.rj.gov.br/sisgam

(8) TemaTres, https://www.vocabularyserver.com/.

(9) Por exemplo, em bibliotecas específicas como https://www.lib.uoguelph.ca/find/find-type-resource, https:// www.lib.usf.edu/library-research/library-collections/, https:// www.ifla.org/publications/node/8968, https://www.ifla.org/ publications/isbd-area-0-content-form-and-media-type-are a, https://libguides.occc.edu/about/collectiondevelopment.

(10)http://thesaurusonline.museus.ul.pt/

\section{Referências}

Baker, L. R. (2004). The Ontology of Artifacts. // Philosophical Explorations. 7 (2004) 99-112. http://people.umass.edu/ Irb/files/bak04ontM.pdf

Berg, J. (1982). Aristotle's theory of definition. // ATTI del convegno internazionale distoria della logica, San Giminiano, 4-8 Decembr, 1982. CLUEB, Bologna, 1983. 19-30.

Bizer, Christian; Heath, Tom; Berners-Lee, Tim. (2009). Linked data - the story so far. Special Issue on Linked Data, International Journal on Semantic Web and Information Systems (IJSWIS), 2009.

Borgo, S; . et al. (2014). Technical artifacts: An integrated perspective. // Applied Ontology. 9 (2014) 217-235.

Brier, S. (2016). O que é a Documentação. Brasília, Briquet de Lemos Livros, 2016.

British Museum Materials Thesauros. (1997). htttp://www.collectionconstruct.org.uk/assets/thesaurus bmm/matintro .htm (2015-05-01)

Cavalcanti, M. L. V. de C.; Fonseca, M. C. L. (2008). Patrimônio imaterial no Brasil: legislação e políticas estaduais. Brasília: UNESCO, 2008.

The CIDOC conceptual reference model. 2014. Version 5.1.12. (2014). ICOM/CIDOC. http://www.cidoc-crm.org/ docs/cidoc_crm_version_5.1.2.pdf.

Collections Trust, Terminologies. https://collectionstrust.org. uk/terminologies/ (2014-09-11).

Broughton, V. (2006). The need for a faceted classification as the basis of all methods of information retrieval. // Aslib Proceedings. 58:1/2 (2006) 49-72

Dahlberg, I. Conceptual definitions for INTERCONCEPT. (1981). // International Classification. (1981) 16-228.

Doerr, M., Bekiari, C., LeBoeuf, P. (2008, September). FRBRoo, a conceptual model for performing arts. // Proceedings of the 2008 Annual Conference of CIDOC, Athens. ICOM/CIDOC, 2008.

Dublin Core Metadata Initiative. http://dublincore.org/.

Generous Interfaces. Europeana Tech. 11 https://pro.europeana.eu/page/issue-11-generous-interfaces (2019-0108).

Europeana. (2017). Definition of Europeana Data Model, version 5.2.7. https://pro.europeana.eu/files/Europeana_Pro fessional/Share your data/Technical requirements/ED M_Documentation/EDM_Definition_v5.2.8_102017.pdf.

Ferrez, H. D., Bianchini, M. H. S. (1987). THESAURUS para acervos museológicos. Rio de Janeiro, Brasil: Fundação Nacional Pró-Memória, 1987.

Fink, E. E (2018). American Art Collaborative (AAC) Linked Open Data (LOD) Initiative. [s.I.]: [s.n.], 2018. http://americanartcollaborative.org/ (2018-02-27).

The Getty Research Institute. Art \& Architecture Thesaurus. http://www.getty.edu/research/tools/vocabularies/aat/index.html (2018-03-18)
Guarino, N. (1995). The Ontological Level. R. Casati, // B. Smith and G. White (eds.). Proceedings of The Philosophy and the Cognitive Science. Vienna: Hölder-PichlerTempsky, 1995.

Guarino, N., Welty, C. (2000). Identity, unity, and individuality: Towards a formaltoolkit for ontological analysis. // Proceedings of The 14th European Conference on Artificial Intelligence. Berlin, Germany: IOS Press, 2000. 219-223. htttp://pdf.aminer.org/000/165/249/identity_unity_and_individuality_towards_a_formal_toolkit_for_ontological.pdf.

Heritage, Canada. (2016). Adopting a Classification System for Collections of Cultural Objects: A Comparison of Nomenclature 4.0 and the Parks Canada Classification System. https://www.canada.ca/en/heritage-information-network/services/collections-documentation-standards/adop ting-classification-system-collections.html (2017-07-09).

Hilpinen, R. (2011). Artifact. E. N. Zalta (Ed.), The Stanford Encyclopedia of Philosophy, 2011. http://stanford.edu/archives/win2011/entries/artifact (2015-06-23).

IFLA. (1998). "Study Group on Functional Requirements for Bibliographic Records: final report". München: K. G. Saur, 1998. (UBCIM Publications New Series).

International Council on Archives. Experts Group on Archival Description (2016). Records in context a conceptual model for archival description. (Consultation Draft v0.1). ICA, 2016.

Marcondes, C. H. (2017). Publicando e interligando acervos digitais na Web através das tecnologias de dados abertos interligados. // Revista Brasileira de Biblioteconomia e Documentação, 13 (2017).

Marcondes, C. H. (2018). Culturally relevant relationships: publishing and connecting digital objects in collections of archives, libraries, and museums over the Web. // Proceedings of The 15th ISKO International Conference, Porto, Portugal, 2018. Porto: Universidade do Porto, 2018.

Marcondes, C. H. (2018). Relacionamentos culturalmente relevantes para interligar objetos do patrimônio digital na Web usando as tecnologias de Dados Abertos Interligados. // Proceedings of The XIX ENANCIB, Londrina, UEL, 2018. Londrina: ANCIB, UEL, 2018.

Marcondes, C. H.; et al. (2016). Proposal of a General Classification Schema for Museum Objects. // Proceedings of The International ISKO Conference 27-29 September 2016, Rio de Janeiro, Brazil. Alemanha: ERGON VERLAG, 2016. 350-358.

Marcondes, C. H.; Souza, E. M. (2018). Vocabulários e acesso integrado a acervos digitais em Memória e Cultura. // Proceedings of The Seminário Internacional de Políticas Culturais, Rio de Janeiro, 9, maio, 2018. Rio de Janeiro: Fundação Casa de Rui Barbosa, 2018. ISBN 978-85-7004-383-2. 109-124.

Ménard, E.; Mas, S.; Alberts, I. (2010). Faceted classification for museum artefacts: A methodology to support web site development of large cultural organizations. // Aslib Proceedings. 62:4/5 (2010).

Merlot, M. (2004). Qu'est-ce qu'un objet patrimonial? // Bulletin des bibliothèques de France. 5. BBF, 2004. 5-10.

Motta, Dilza (2006). Tesauro de Cultura Material dos Índios no Brasil.Rio de Janeiro, Museu do Índio, 2006.

Nomenclature for Museum Cataloging. (2018). http://www.nomenclature.info/apropos-about.app?lang=en.

Perfoming Arts. Wikipedia. https://en.wikipedia.org/wiki/Performing_arts (2018-06-14).

Searle, John. (1995). The construction of social reality. New York, The Free Press, 1995.

Sinclair, P. A. S.; et al. (2005). Concept browsing for multimedia retrieval in the SCULPTEUR project. 
SKOS Simple Knowledge Organization System Primer (2009). W3C. https://www.w3.org/TR/skos-primer/

Stransky, Z. Z. (1985). Originals versus substitutes'. // V. Sofka (ed.). Originals and substitutes in museum. Stockholm: ICOFOM. 95-102. ICOFOM Study Series 9.

Tesauro de Folclore e Cultura Popular. http://www.cnfcp.gov. br/interna.php?ID_Secao=30 (2016-03-05).

Tesauro de Objetos do Patrimônio Cultural nos Museus Brasileiros. http://www.tesauromuseus.com.br/ (2017-0522).

\section{Anexo 1. Representação do esquema proposto em SKOS}

Thesaurus de Acervos Científicos em língua portuguesa. http://thesaurusonline.museus.ul.pt/hierarquica.aspx (2015-07-21).

UNESCO. Intagible Heritage. http://www.unesco.org/new/pt/ brasilia/culture/world-heritage/intangible-heritage/ (201604-23)

Whitelaw, M. (2015). Generous interfaces for digital cultural collections. Digital Humanities Quarterly, 9:1 (2015).

Wikipedia. Performing Arts. https://en.wikipedia.org/wiki/Performing_arts.

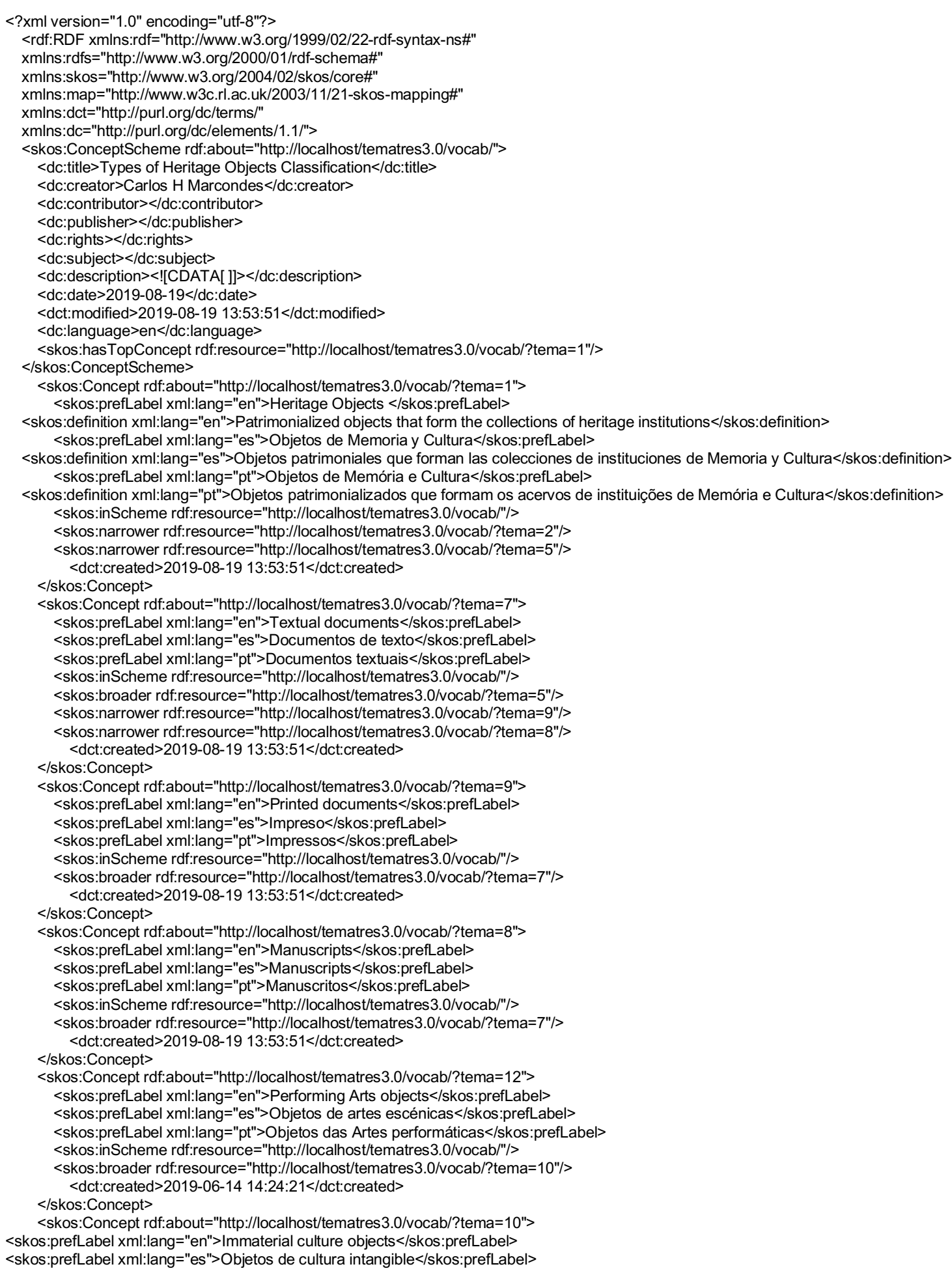


<skos:prefLabel xml:lang="pt">Objetos de cultura imaterial</skos:prefLabel> <skos:inScheme rdf:resource="http://localhost/tematres3.0/vocab/"/>

<skos:broader rdf:resource="http://localhost/tematres3.0/vocab/?tema=5"/>

<skos:narrower rdf:resource="http://localhost/tematres3.0/vocab/?tema=12"/>

<skos:narrower rdf:resource="http://localhost/tematres3.0/vocab/?tema=11"/>

$<$ dct:created>2019-08-19 13:53:51</dct:created>

$</$ skos:Concept>

<skos:Concept rdf:about="http://localhost/tematres3.0/vocab/?tema=6">

<skos:prefLabel xml:lang="en">Material Culture objects</skos:prefLabel>

<skos:definition xml:lang="en">Material Culture objects or artefacts (the 15 Categories of Ferrez and Bianchini (1987) Thesaurus except the 16th Category,

Samples/Fragments) </skos:definition>

<skos:prefLabel xml:lang="es">Objetos de cultura material o artefactos</skos:prefLabel>

<skos:definition xml:lang="es">Objetos que componen las 15 Categorías del Tesauro de Feresa y Bianchini (1987) excepto la 16 Categoría, Muestras / Fragmentos </skos:definition>

<skos:prefLabel xml:lang="pt">Objetos de cultura material ou Artefatos</skos:prefLabel>

<skos:definition xml:lang="pt">Objetos que constituem as 15 Categorias do Thesaurus de Ferrez e Bianchini (1987) exceto a 16 Categoria, Amostras/fragmentos</skos:definition>

<skos:inScheme rdf:resource="http://localhost/tematres3.0/vocab/"/>

<skos:broader rdf:resource="http://localhost/tematres3.0/vocab/?tema=5"/>

<skos:exactMatch rdf:resorce="http://www.cidoc-crm.org/html/5.0.4/cidoc-crm.htm|\#E71"/> <skos:exactMatch rdf:resorce="http://vocab.getty.edu/aat/300386957"/> $<$ dct:created>2019-08-19 13:53:51</dct:created>

$<$ skos:Concept>

<skos:Concept rdf:about="http://localhost/tematres3.0/vocab/?tema=3"> <skos:prefLabel xml:lang="en">Inorganic objects</skos:prefLabel>

<skos:definition xml:lang="en">Heritage objects that are inorganic, such as mineralogy collections</skos:definition> <skos:prefLabel xml:lang="es">Objetos inorgánicos</skos:prefLabel>

<skos:definition xml:lang="es">Objetos patrimoniales inorgánicos, como colecciones de mineralogía</skos:definition> <skos:prefLabel xml:lang="pt">Objetos inorgânicos</skos:prefLabel>

<skos:definition xml:lang="pt">Objetos patrimonializados que são inorgânicos, como coleções de mineralogia</skos:definition> <skos:inScheme rdf:resource="http://localhost/tematres3.0/vocab/"/> <skos:broader rdf:resource="http://localhost/tematres3.0/vocab/?tema=2"/> <dct:created>2019-08-19 13:53:51</dct:created>

$</$ skos:Concept>

<skos:Concept rdf:about="http://localhost/tematres3.0/vocab/?tema=2"> <skos:prefLabel xml:lang="en">Natural objects</skos:prefLabel>

<skos:definition xml:lang="en">Heritage objects that are originally organic, such as fossils, herbariums, zoos, entomological collections</skos:definition> <skos:prefLabel xml:lang="es">Objetos naturales</skos:prefLabel>

<skos:definition xml:lang="es">Objetos patrimoniales que son originalmente orgánicos, como fósiles, herbarios, zoológicos, colecciones entomológicas</skos:definition>

<skos:prefLabel xml:lang="pt">Objetos naturais</skos:prefLabel>

<skos:definition xml:lang="pt">Objetos patrimoniais originalmente orgânicos, como fósseis, herbários, zoológicos, coleções entomológicas</skos:definition> <skos:inScheme rdf:resource="http://localhost/tematres3.0/vocab/"/>

<skos:broader rdf:resource="http://localhost/tematres3.0/vocab/?tema=1"/>

<skos:narrower rdf:resource="http://localhost/tematres3.0/vocab/?tema=3"/>

<skos:narrower rdf:resource="http://localhost/tematres3.0/vocab/?tema=4"/> <dct:created>2019-06-14 14:24:21</dct:created>

$<$ skos:Concept>

<skos:Concept rdf:about="http://localhost/tematres3.0/vocab/?tema=4">

<skos:prefLabel xml:lang="en">Organic objects</skos:prefLabel>

$<$ skos:prefLabel xml:lang="es">Objetos orgánicos</skos:prefLabel>

<skos:prefLabel xml:lang="pt">Objetos orgânicos</skos:prefLabel>

<skos:inScheme rdf:resource="http://localhost/tematres3.0/vocab/"/>

<skos:broader rdf:resource="http://localhost/tematres3.0/vocab/?tema=2"/>

<dct:created>2019-08-19 13:53:51</dct:created>

$</$ skos:Concept>

<skos:Concept rdf:about="http://localhost/tematres3.0/vocab/?tema=11">

<skos:prefLabel xml:lang="en">Folk Culture Perfoming objects</skos:prefLabel>

<skos:prefLabel xml:lang="es">Objetos de performance de la cultura popular</skos:prefLabel>

<skos:prefLabel xml:lang="pt">Objetos performáticos da Cultura popular</skos:prefLabel>

<skos:inScheme rdf:resource="http://localhost/tematres3.0/vocab/"/>

<skos:broader rdf:resource="http://localhost/tematres3.0/vocab/?tema=10"/>

$<$ dct:created>2019-08-19 13:53:51</dct:created>

$</$ skos:Concept>

<skos:Concept rdf:about="http://localhost/tematres3.0/vocab/2tema=5">

<skos:prefLabel xml:lang="en">Human Culture products - man-made objects</skos:prefLabel>

<skos:prefLabel xml:lang="es">Productos de cultura humana - Objetos creados por el hombre</skos:prefLabel>

<skos:prefLabel xml:lang="pt">Produtos da Cultura Humana - objetos feitos pelo homem</skos:prefLabel>

<skos:inScheme rdf:resource="http://localhost/tematres3.0/vocab/"/>

<skos:broader rdf:resource="http://localhost/tematres3.0/vocab/?tema=1"/>

<skos:narrower rdf:resource="http://localhost/tematres3.0/vocab/?tema=7"/>

<skos:narrower rdf:resource="http://localhost/tematres3.0/vocab/?tema=10"/>

<skos:narrower rdf:resource="http://localhost/tematres3.0/vocab/?tema=6"/>

<dct:created>2019-08-19 13:53:51</dct:created>

$</$ skos:Concept $>$

$<$ /rdf:RDF>

Enviado: 2019-03-30. Segunda versión: 2019-08-26 Aceptado: 2019-09-03. 